\title{
752.
}

\section{ON THE FINITE GROUPS OF LINEAR TRANSFORMATIONS OF}

\section{A VARIABLE; WITH A CORRECTION.}

[From the Mathematische Annalen, t. xvI. (1880), pp. 260-263; 439, 440.]

IN the paper "Ueber endliche Gruppen linearer Transformationen einer Veränderlichen," Math. Ann. t. xII. (1877), pp. 23-46, Prof. Gordan gave in a very elegant form the groups of 12,24 and 60 homographic transformations $\frac{a x+b}{c x+d}$. The groups of 12 and 24 are in the like form, the group of 24 thus containing as part of itself the group of 12 ; but the group of 60 is in a different form, not containing as part of itself the group of 12. It is, I think, desirable to present the group of 60 in the form in which it contains as part of itself Gordan's group of 12: and moreover to identify the group of 60 with the group of the 60 positive permutations of 5 letters: or (writing $a b c$ for the cyclical permutation $a$ into $b, b$ into $c, c$ into $a$, and so in other cases) say with the group of the 60 positive permutations $1, a b c, a b . c d$ and abcde.

Any two forms of a group are, it is well known, connected as follows, viz. if $1, \alpha, \beta, \ldots$ are the functional symbols of the one form, then those of the other form are $1,9 \alpha 9^{-1}, 9 \beta 9^{-1}, \ldots$ (where in the case in question 9 is a functional symbol of the like homographic form, $\left.9 x=\frac{A x+B}{C x+D}\right)$. But instead of obtaining the new form in this manner, $I$ found it easier to use the values of the rotation-symbol

$$
\cos \frac{\pi}{q}+\sin \frac{\pi}{q}(i \cos X+j \cos Y+k \cos Z)
$$

for the axes of the icosahedron or dodecahedron, given in my paper "Notes on polyhedra," Quart. Math. Jour. t. vII. (1866), pp. 304-316, [375]; viz. if for any axes, $\lambda, \mu, \nu$ denote the parameters of rotation $\tan \frac{\pi}{q} \cos X, \tan \frac{\pi}{q} \cos Y, \tan \frac{\pi}{q} \cos Z$, then, 
by a formula which is in fact equivalent to that given in my note "On the correspondence of Homographies and Rotations," Math. Annalen, t. xv. (1879), pp. 238-240, [660], the corresponding homographic function of $x$ is

where $i$ denotes $\sqrt{-1}$ as usual.

$$
\frac{(-\nu-i) x+\lambda+i \mu}{(\lambda-i \mu) x+\nu-i}
$$

The new formulæ for the group of 60 , or icosahedron group, of homographic functions $\frac{\alpha x+\beta}{\gamma x+\delta}$ are contained in the following table, where the four columns show the values of the coefficients $\alpha, \beta, \gamma, \delta$ respectively: and where in the outside column, the substitution is represented as a permutation-symbol on the five letters abcde: moreover for shortness $\Theta$ is written to denote $\sqrt{5}$.

The Group of 60.

\begin{tabular}{|c|c|c|c|c|c|}
\hline & $a$ & $\beta$ & $\gamma$ & $\delta$ & \\
\hline 1 & 1 & 0 & 0 & 1 & 1 \\
\hline 2 & -1 & 0 & 0 & 1 & $a b \cdot c d$ \\
\hline 3 & 0 & 1 & 1 & 0 & $a c \cdot b d$ \\
\hline 4 & 0 & -1 & 1 & 0 & $a d \cdot b c$ \\
\hline 5 & 2 & $-3+\theta+i(\quad 1-\theta)$ & $-3+\theta+i(-1+\theta)$ & -2 & $b c \cdot d e$ \\
\hline 6 & 2 & $-3+\theta+i(-1+\theta)$ & $-3+\theta+i(1-\theta)$ & -2 & $a e \cdot b c$ \\
\hline 7 & 2 & $3-\theta+i(-1+\theta)$ & $3-\theta+i(1-\theta)$ & -2 & $a d . c e$ \\
\hline 8 & 2 & $3-\theta+i(1-\theta)$ & $3-\theta+i(-1+\theta)$ & -2 & $a d . b e$ \\
\hline 9 & 2 & $-1-\theta+i(1-\theta)$ & $-1-\theta+i(-1+\theta)$ & -2 & $a e \cdot c d$ \\
\hline 10 & 2 & $-1-\theta+i(-1+\theta)$ & $-1-\theta+i(1-\theta)$ & -2 & $a b . d e$ \\
\hline 11 & 2 & $1+\theta+i(-1+\theta)$ & $1+\theta+i(1-\theta)$ & -2 & $b e . c d$ \\
\hline 12 & 2 & $1+\theta+i(1-\theta)$ & $1+\theta+i(-1+\theta)$ & -2 & $a b \cdot c e$ \\
\hline 13 & 2 & $-1-\theta+i(-3-\theta)$ & $-1-\theta+i(3+\theta)$ & -2 & $a c . b e$ \\
\hline 14 & 2 & $-1-\theta+i(3+\theta)$ & $-1-\theta+i(-3-\theta)$ & -2 & $b d . c e$ \\
\hline 15 & 2 & $1+\theta+i(\quad 3+\theta)$ & $1+\theta+i(-3-\theta)$ & -2 & $a e \cdot b d$ \\
\hline 16 & 2 & $1+\theta+i(-3-\theta)$ & $1+\theta+i(\quad 3+\theta)$ & -2 & $a c . d e$ \\
\hline 17 & $-i$ & $i$ & 1 & 1 & $a b c$ \\
\hline 18 & -1 & $i$ & 1 & $i$ & $a c b$ \\
\hline 19 & 1 & $-i$ & 1 & $i$ & $a d c$ \\
\hline 20 & $-i$ & $-i$ & 1 & -1 & acd \\
\hline 21 & $i$ & $i$ & 1 & -1 & $\overline{a d b}$ \\
\hline 22 & 1 & $i$ & 1 & $-i$ & $a b d$ \\
\hline 23 & -1 & $-i$ & 1 & $-i$ & $b c d$ \\
\hline 24 & $i$ & $-i$ & 1 & 1 & $b d c$ \\
\hline
\end{tabular}


$a$

$\beta$

$\gamma$

$\delta$

\begin{tabular}{|c|c|c|c|c|c|}
\hline 25 & $-1-\theta+i(\quad 3+\theta)$ & 2 & -2 & $-1-\theta+i(-3-\theta)$ & aec \\
\hline 26 & $1+\theta+i(3+\theta)$ & 2 & -2 & $1+\theta+i(-3-\theta)$ & ace \\
\hline 27 & $1+\theta+i(-3-\theta)$ & 2 & -2 & $1+\theta+i(3+\theta)$ & bed \\
\hline 28 & $-1-\theta+i(-3-\theta)$ & 2 & -2 & $-1-\theta+i(3+\theta)$ & bde \\
\hline 29 & $-3+\theta+i(1-\theta)$ & 2 & 2 & $3-\theta+i(1-\theta)$ & bec \\
\hline 30 & $-3+\theta+i(-1+\theta)$ & 2 & 2 & $3-\theta+i(-1+\theta)$ & bce \\
\hline 31 & $3-\theta+i(-1+\theta)$ & 2 & 2 & $-3+\theta+i(-1+\theta)$ & aed \\
\hline 32 & $3-\theta+i(1-\theta)$ & 2 & 2 & $-3+\theta+i(1-\theta)$ & ade \\
\hline 33 & 2 & $-1-\theta+i(-1+\theta)$ & $1+\theta+i(-1+\theta)$ & & cde \\
\hline 34 & 2 & $1+\theta+i(1-\theta)$ & $-1-\theta+i(1-\theta)$ & & ced \\
\hline 35 & 2 & $-1-\theta+i(1-\theta)$ & $1+\theta+i(1-\theta)$ & & $a e b$ \\
\hline 36 & 2 & $1+\theta+i(-1+\theta)$ & $-1-\theta+i(-1+\theta)$ & & abe \\
\hline 37 & $-1-\theta+i(-3-\theta)$ & 2 & 2 & $1+\theta+i(-3-\theta)$ & abcde \\
\hline 38 & $-1-\theta+i(1-\theta)$ & 2 & 2 & $1+\theta+i(1-\theta)$ & $a c e b d$ \\
\hline 39 & $-1-\theta+i(-1+\theta)$ & 2 & 2 & $1+\theta+i(-1+\theta)$ & adbec \\
\hline 40 & $-1-\theta+i(3+\theta)$ & 2 & 2 & $1+\theta+i(3+\theta)$ & $a e d c b$ \\
\hline 41 & $1+\theta+i(3+\theta)$ & 2 & 2 & $-1-\theta+i(3+\theta)$ & adceb \\
\hline 42 & $1+\theta+i(-1+\theta)$ & 2 & 2 & $-1-\theta+i(-1+\theta)$ & acbde \\
\hline 43 & $1+\theta+i(1-\theta)$ & 2 & 2 & $-1-\theta+i(1-\theta)$ & $a e d b c$ \\
\hline 44 & $1+\theta+i(-3-\theta)$ & 2 & 2 & $-1-\theta+i(-3-\theta)$ & abecd \\
\hline 45 & $-1-\theta+i(-1+\theta)$ & 2 & -2 & $-1-\theta+i(1-\theta)$ & acbed \\
\hline 46 & $-3+\theta+i(-1+\theta)$ & 2 & -2 & $-3+\theta+i(1-\theta)$ & $a b d c e$ \\
\hline 47 & $3-\theta+i(-1+\theta)$ & 2 & -2 & $3-\theta+i(1-\theta)$ & $a e c d b$ \\
\hline 48 & $1+\theta+i(-1+\theta)$ & 2 & -2 & $1+\theta+i(1-\theta)$ & $a d e b c$ \\
\hline 49 & $1+\theta+i(\quad 1-\theta)$ & 2 & -2 & $1+\theta+i(-1+\theta)$ & $a e c b d$ \\
\hline 50 & $3-\theta+i(1-\theta)$ & 2 & -2 & $3-\theta+i(-1+\theta)$ & acdeb \\
\hline 51 & $-3+\theta+i(1-\theta)$ & 2 & -2 & $-3+\theta+i(-1+\theta)$ & abedc \\
\hline 52 & $-1-\theta+i(\quad 1-\theta)$ & 2 & -2 & $-1-\theta+i(-1+\theta)$ & adbce \\
\hline 53 & 2 & $-3+\theta+i(-1+\theta)$ & $3-\theta+i(-1+\theta)$ & 2 & $a e b d c$ \\
\hline 54 & 2 & $-1-\theta+i(3+\theta)$ & $1+\theta+i(3+\theta)$ & 2 & abced \\
\hline 55 & 2 & $1+\theta+i(-3-\theta)$ & $-1-\theta+i(-3-\theta)$ & 2 & $a d e c b$ \\
\hline 56 & 2 & $3-\theta+i(1-\theta)$ & $-3+\theta+i(\quad 1-\theta)$ & 2 & acdbe \\
\hline 57 & 2 & $-3+\theta+i(1-\theta)$ & $3-\theta+i(1-\theta)$ & 2 & $a b d e c$ \\
\hline 58 & 2 & $-1-\theta+i(-3-\theta)$ & $1+\theta+i(-3-\theta)$ & 2 & adcbe \\
\hline 59 & 2 & $1+\theta+i(3+\theta)$ & $-1-\theta+i(3+\theta)$ & 2 & aebcd \\
\hline 60 & 2 & $3-\theta+i(-1+\theta)$ & $-3+\theta+i(-1+\theta)$ & 2 & $a c e d b$ \\
\hline
\end{tabular}


This contains (as one of five groups of 12) the group of the positive permutations of abcd; and, completing this into a group of 24 , we have

Groups of 12 AND 24.

\begin{tabular}{|c|c|c|c|c|c|}
\hline 1 & 1 & 0 & 0 & 1 & 1 \\
\hline 2 & -1 & 0 & 0 & 1 & $a b \cdot c d$ \\
\hline 3 & 0 & 1 & 1 & 0 & $a c . b d$ \\
\hline 4 & 0 & -1 & 1 & 0 & $a d . b c$ \\
\hline 5 & $-i$ & $i$ & 1 & 1 & $a b c$ \\
\hline 6 & -1 & $i$ & 1 & $i$ & $a c b$ \\
\hline 7 & 1 & $-i$ & 1 & $i$ & $a d c$ \\
\hline 8 & $-i$ & $-i$ & 1 & -1 & acd \\
\hline 9 & $i$ & $i$ & 1 & -1 & $a d b$ \\
\hline 10 & 1 & $i$ & 1 & $-i$ & $a b d$ \\
\hline 11 & -1 & $-i$ & 1 & $-i$ & $b c d$ \\
\hline 12 & $i$ & $-i$ & 1 & 1 & $b d c$ \\
\hline 13 & $i$ & 0 & 0 & 1 & $a d b c$ \\
\hline 14 & $-i$ & 0 & 0 & 1 & $a c b d$ \\
\hline 15 & 0 & $i$ & 1 & 0 & $c d$ \\
\hline 16 & 0 & $i$ & -1 & 0 & $a b$ \\
\hline 17 & 1 & -1 & 1 & 1 & $a c d b$ \\
\hline 18 & $-i$ & -1 & 1 & $i$ & $b d$ \\
\hline 19 & $i$ & 1 & 1 & $i$ & abcd \\
\hline 20 & 1 & 1 & 1 & -1 & $b c$ \\
\hline 21 & -1 & -1 & 1 & -1 & $a b d c$ \\
\hline 22 & $i$ & -1 & 1 & $-i$ & $a c$ \\
\hline 23 & $-i$ & 1 & 1 & $-i$ & $a d c b$ \\
\hline 24 & -1 & 1 & 1 & 1 & $a d$ \\
\hline
\end{tabular}

The groups of 60 and 24 thus each of them contain the group of 12,

$$
\pm x, \pm \frac{1}{x}, \pm i \frac{1-x}{1+x}, \pm i \frac{1+x}{1-x}, \pm \frac{x+i}{x-i}, \pm \frac{x-i}{x+i} .
$$

It may be remarked that, to verify the periodicities of the forms contained in the group of 60 , we have as the conditions that

$\frac{\alpha x+\beta}{\gamma x+\delta}$ may be periodic of the order $2, \frac{(\alpha+\delta)^{2}}{\alpha \delta-\beta \gamma}=0$, that is, $\alpha+\delta=0$,

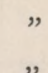

$3, \quad, \quad=1$,

$5, \quad, \quad=\frac{1}{2}(3+\sqrt{5})$. 
For instance, in the form

we have

$$
\frac{[-1-\Theta+i(-3-\Theta)] x+2}{2 x+[1+\Theta+i(-3-\Theta)]}
$$

and therefore

$$
\begin{gathered}
\alpha \delta=-(1+\Theta)^{2}-(3+\Theta)^{2}, \quad=-20-8 \Theta, \quad \beta \gamma=4, \\
\alpha+\delta=-2 i(3+\Theta):
\end{gathered}
$$

as it should be.

$$
\frac{(\alpha+\delta)^{2}}{\alpha \delta-\beta \gamma}=\frac{-4(3+\Theta)^{2}}{-8(3+\Theta)}, \quad=\frac{3+\Theta}{2}=\frac{1}{2}(3+\sqrt{5})
$$

Cambridge, 11 Nov. 1879.

Correction*, pp. $439,440$.

I erroneously assumed that the symbol $a d c b$ could be taken as corresponding to the linear transformation $i x$ : but this was obviously wrong, for it gave $b d$ as corresponding to the transformation $-i x$, and these are not of the same order, but of the orders 4 and 2 respectively. The proper symbol is $a d b c$, as given above, and the remaining eleven symbols are then at once obtained.

Cambridge, 17 Feb. 1880.

[* The correction in the Table of the Groups of 12 and 24 has been inserted in the Table as now printed on p. 240 ; it applies to the second half of the column of symbols on the extreme right-hand.] 